\title{
MS03-04 | Biophysical Methods to Aid Protein Crystallization in A Pharmaceutical Setting
}

Öster, Linda (AstraZeneca, Mölndal, SWE)

In the Protein Structure group at AstraZeneca, the overall goal is to support projects with structures. A key success factor is the quality control of the proteins of interest using a range of techniques including mass spectrometry, size exclusion chromatography and gel electrophoresis. The goal is to ensure high purity and homogeneity before entering crystallization trials. To learn more about the protein and further verify the quality we often use Dynamic Light Scattering and Thermal shift assay and these methods are also used to identify the best formulation.

As the great majority of our structures are protein-compound structures we are highly dependent on biophysical characterization of the protein-compound interaction. The purpose is to verify that we work on compounds that show specific binding, to rank the different compounds and identify differences in binding mode to further guide our crystallization trials. This is particularly important in a project during the hit identification campaign when many compounds from different sources are considered interesting but could be false positives. The methods used vary, and include Nuclear Magnetic Resonance, Surface Plasmon Resonance, Isothermal Titration Calorimetry and Thermal Shift Assay, and often several methods are in use for one project. Furthermore, we have seen that it can be very powerful to use biophysical methods to ensure compound binding to our specific crystallization construct and/or in the actual soaking/co-crystallization condition.

We will present our way of working including different project examples on how biophysical techniques have aided our efforts to support projects with structures. 